\title{
Influence of 2,5-dichloro-1,4-benzoquinone on jack bean urease activity. Inhibitory effect, total reducing capacity and DPPH radical scavenging activity
}

\author{
Mirosława Kot ${ }^{\bowtie}$ and Zofia Olech \\ Jagiellonian University, Faculty of Chemistry, Kraków, Poland
}

\begin{abstract}
Inhibition of jack bean activity by 2,5-dichloro-1,4-benzoquinone (DCBQ) was studied in phosphate buffer, $\mathrm{pH}$ 7.0. It was found that DCBQ acted as a strong, time and concentration dependent inactivator of urease. Under the experimental conditions obeyed the terms of pseudo-first-order reaction, urease was totally inactivated. Application of Wilson-Kitz method proved that the urease-DCBQ interaction followed a simple bimolecular process and the presence of intermediate complex was undetectable. The determined second order rate constant of the inactivation was $0.053(\mu \mathrm{M} \min )^{-1}$. Thiols such as L-cysteine, glutathione and dithiothreitol (DTT) protected urease from inhibition by $D C B Q$ but $D C B Q-$ modified urease did not regain its activity after DTT application. The thiol protective studies indicated an essential role of urease thiol(s) in the inhibition. The irreversibility of the inactivation showed that the process was a result of a direct modification of urease thiol(s) by DCBQ (DCBQ chlorine(s) substitution). The decomposition of DCBQ in aqueous solution at natural light exposure was monitored by visible spectrophotometry, determination of the total reducing capacity (Folin-Ciocalteu method) and DPPH (2,2-diphenyl-1-picrylhydrazyl) radical scavenging ability. The DCBQ conversion resulted in a decrease of the inhibition power and was well correlated with the increase of the total reducing capacity and DPPH scavenging ability. These findings were attributed to DCBQ transformation by photolysis and the hydrolysis effect was found to be negligible.
\end{abstract}

Keywords: urease, inhibition, 2,5-dichloro-1,4-naphthoquinone, DPPH scavenging activity, reducing capacity, Folin-Ciocalteu method

Received: 31 August, 2011; revised: 23 November, 2011; accepted: 12 December, 2011; available on-line: 15 December, 2011

\section{INTRODUCTION}

Urease (urea amidohydrolase, EC 3.5.1.5) is a ubiquitous enzyme catalyzing the hydrolysis of urea: $\mathrm{CO}\left(\mathrm{NH}_{2}\right)_{2}+\mathrm{H}_{2} \mathrm{O} \stackrel{\text { urease }}{\longrightarrow} 2 \mathrm{NH}_{3}+\mathrm{CO}_{2}$. The sources of the enzyme are plants, algae, fungi and bacteria (Mobley \& Hausinger, 1989; Sirko \& Brodzik, 2000; Krajewska, 2009). The best known urease is that obtained from jack bean seeds. Jack bean urease is a homohexamer (alpha ${ }_{6}$ ). Its active site contains two nickel ions directly involved in binding of substrates and inhibitors (Karplus \& Pearson, 1997; Benini et al., 1999). Moreover, the urease catalytic activity strongly depends on its thiol residues. The total number of thiol groups is 15 per subunit. One of them, cysteine-592, is located on a mobile flap closing the active site of urease. Chemical modification of cysteine-592 results in inactivation of the enzyme (Mamiya et al., 1985; Norris \& Brocklehurst, 1976). Therefore, numerous of sulphide compounds as well as thiol-reactive reagents display inhibitory effect on urease (Mobley \& Hausinger, 1989; Kot et al., 2000; Kot \& Bicz, 2008). Urease is subject of numerous medicinal, agricultural and environmental studies. Many of them focus on the prevention of the harmful urease activity for human and animal health. The aim of these investigations is a search for effective urease inhibitors. The interesting group of chemicals examined in terms of blocking the enzyme activity are quinones (Bundy \& Bremner, 1973; Ashiralieva \& Kleiner, 2003; Zaborska et al., 2007). Quinones are compounds of wide occurrence in nature thus they can be obtained from natural sources. 5-hydroxy-1,4naphthoquinone (juglone) is a brown dye isolated from fruits, shells and leaves of walnut trees (Juglans). The main source of 2-hydroxy-1,4-naphthoquinone (lawsone) is the henna herb (Lawsonia inermis L.). 1,2-dihydroxyanthraquinone (alizarin) occurs in the root of the common madder (Rubia tinctorum) and in various parts of Indian madder (Rubia cordifolia) (Dweek, 2002; Briton, 2006). Vitamin $\mathrm{K}$ group posses the 2-methyl-1,4-naphthoquinone structure. In biological systems quinones act mainly as electron carriers in photosynthetic and respiratory electron transport chains. Another feature that makes quinones promising inhibitors is their high reactivity. Quinones are electrophiles and therefore can covalently modify of cellular nucleophiles such as glutathione or cysteine residues. Moreover, some quinones are potent redox molecules. They act as catalysts in the production of ROS (reactive oxygen species) such as superoxide anion radicals, hydrogen peroxide and hydroxyl radicals (Bolton et al., 2000; Rodriguez et al., 2005). ROS are responsible for damage of macromolecules. This diversity of quinone mechanisms broadens the target of their action. Some quinones act accordingly to one of two possible ways, others are thought to display both mechanisms simultaneously (Zaborska et al., 2007; 2009). On the other hand, the high reactivity of quinones results in their low resistance to chemical deterioration mainly due to hydrolysis and photolysis (Ononye et al., 1986; Sarr et al., 1995). Extensive investigations have already resulted in finding potent inhibitors among quinones e.g.

e-mail: kot@chemia.uj.edu.pl

Abbreviations: DCBQ, 2,5-dichloro-1,4-benzoquinone; DPPH, 2,2-diphenyl-1-picrylhydrazyl; DTT, dithiothreitol; ROS, reactive oxygen species 
chloranil (tetrachloro-1,4-benzoquinone) and dichlone (2,3-dichloro-1,4-naphthoquinone) which are the most frequently used fungicides (Lukens, 1971).

The purpose of this work was to study a less known urease inhibitor 2,5-dichloro-1,4-benzoquinone (DCBQ) and elucidate the kinetics and mechanism of urease inhibition by DCBQ. An influence of DCBQ decomposition in aqueous solution on the urease inhibition was observed. The process was studied by monitoring changes in visible spectra, total reducing capacity and DPPH scavenging activity measurements. Photoinduced quinone decomposition is still a subject of investigations. The elucidation of this process should be accompanied by the research presenting the influence of the quinone decomposition on nature, especially if quinone is implemented intentionally into the environment (e.g. as a fungicide). Furthermore, understanding degradation of quinones as toxic compounds may have implications for waste treatment.

\section{MATERIALS AND METHODS}

Materials. Jack bean urease, Sigma type III, specific activity $16 \mathrm{U} \mathrm{mg}^{-1}$ solid, urea (Molecular Biology Reagent), 2,5-dichloro-1,4-benzoquinone (DCBQ), dithiothreitol (DTT), L-cysteine, glutathione and 2,2-diphenyl1-picrylhydrazyl (DPPH) were obtained from Aldrich. Folin-Ciocalteu phenol reagent, $2 \mathrm{~N}$ was from SigmaAldrich. Other chemicals were obtained from POCh, Gliwice, Poland. All reagents used were of analytical grade. Phosphate buffer $20 \mathrm{mM}, \mathrm{pH} 7.0$, was prepared by adjusting $\mathrm{pH}$ of phosphoric $(\mathrm{V})$ acid with $\mathrm{NaOH}$. EDTA $(2 \mathrm{mM})$ was added to all enzyme-containing solutions.

DCBQ stock solution. DCBQ stock solution was obtained by dissolving DCBQ in distilled water. Its concentration was equal to $1.9 \mathrm{mM}$. Less concentrated solutions of $0.95 \mathrm{mM}$ and $12.8 \mu \mathrm{M}$ were prepared by dilution of the initial solution. All stock solutions were stored at ambient temperature and exposured to natural sun light. Control stock solution was stored in the dark, at $5^{\circ} \mathrm{C}$. Changes of the stock solutions were monitored for $288 \mathrm{~h}$. The time when the stock solutions were prepared was pointed as zero time. Kinetic studies used DCBQ solution prepared just before the experiment.

Standard urease activity assay. The standard assay mixture $\left(10 \mathrm{~cm}^{3}\right)$ consisted of $50 \mathrm{mM}$ urea in $20 \mathrm{mM}$ phosphate buffer, pH 7.0 and 2 mM EDTA. The reactions were initiated by the addition of $0.1 \mathrm{~cm}^{3}$ enzymecontaining solution ( $1 \mathrm{mg} \mathrm{cm}^{-3}$ of urease, $20 \mathrm{mM}$ phosphate buffer $\mathrm{pH} 7.0,2 \mathrm{mM}$ EDTA) and the urease activity was determined by measuring ammonia concentration after a 5-min reaction. Ammonia was determined by the spectrophotometric, phenol-hypochlorite method (Weatherburn, 1967). The absorbance was registered in 1-cm quartz cuvette at $630 \mathrm{~nm}$ using a MARCEL MEDIA spectrophotometer. The measurements were performed at ambient temperature. The activity of uninhibited urease was taken as $100 \%$.

Urease inhibition by DCBQ. Kinetic studies. The solution of urease was incubated with the solution of DCBQ in the absence of urea. The incubation mixture contained $0.5 \mathrm{mg} \mathrm{cm}^{-3}$ of urease, $20 \mathrm{mM}$ phosphate buffer, $\mathrm{pH} 7.0,2 \mathrm{mM}$ EDTA and 9.6, 6.4, 5.5 or $4.8 \mu \mathrm{M}$ DCBQ. The time when the enzyme and the inhibitor were mixed was marked as zero time of incuba- tion. After appropriate period of time, aliquots $\left(0.2 \mathrm{~cm}^{3}\right)$ from the incubation mixture were withdrawn and transferred into the standard assay mixtures for urease activity determination.

Influence of DCBQ decomposition in aqueous solution on urease inhibition. The inhibitory power of DCBQ solution was tested for $288 \mathrm{~h}$ every $24 \mathrm{~h}$ or $48 \mathrm{~h}$. DCBQ was incubated for $5 \mathrm{~min}$ with urease. The incubation mixture contained $0.5 \mathrm{mg} \mathrm{cm}^{-3}$ of urease, $20 \mathrm{mM}$ phosphate buffer, $\mathrm{pH}$ 7.0, $2 \mathrm{mM}$ EDTA and $6.4 \mu \mathrm{M}$ DCBQ. After a 5-min incubation, aliquots $\left(0.2 \mathrm{~cm}^{3}\right)$ from the incubation mixture were withdrawn and transferred into the standard assay mixtures for urease activity determination. Control experiments were done using stock DCBQ solution stored in the dark, $5^{\circ} \mathrm{C}$.

Thiol protective experiment. In the protective experiment, all the preincubation mixtures contained 0.5 $\mathrm{mg} \mathrm{cm} \mathrm{cm}^{-3}$ urease, $20 \mathrm{mM}$ phosphate buffer, $\mathrm{pH} \mathrm{7.0,2}$ mM EDTA, 9.6 $\mu \mathrm{M}$ DCBQ (freshly prepared) and 0.25 $\mathrm{mM}$ thiol: dithiothreitol (DT'T), L-cysteine or glutathione. The mixture was incubated for $5 \mathrm{~min}$. After incubation a sample of the preincubation mixture $\left(0.2 \mathrm{~cm}^{3}\right)$ was withdrawn and transferred into the standard assay mixture for urease activity determination.

Reactivation of urease inactivated by DCBQ. The reactivation of DCBQ-inactivated urease was studied by DT'T application. The incubation mixture contained 0.5 $\mathrm{mg} \mathrm{cm}^{-3}$ urease, 9.6 $\mu \mathrm{M}$ DCBQ (freshly prepared), 20 $\mathrm{mM}$ phosphate buffer, $\mathrm{pH}$ 7.0. After a 5-min incubation of urease with the inhibitor, a small volume of DTT solution was added to $50 \mu \mathrm{M}$. The activity of urease was determined before and after the DT'T addition. After appropriate periods of time, samples of the incubation mixture were withdrawn and transferred into the standard assay mixture and urease activity was determined. The experiment was three times repeated. The effect of DT'T addition was also tested for shorter incubation time (1 and 2-min incubation).

Monitoring of DCBQ decomposition in aqueous solution. Visible spectra. Spectra of 1.9-mM DCBQ aqueous stock solution stored at ambient temperature and exposured to natural light were recorded in the wavelength range $400-700 \mathrm{~nm}$ in $5-\mathrm{cm}$ quartz cuvette on a MARCEL MEDIA spectrophotometer. The stock solution was monitored for $288 \mathrm{~h}$. Control spectra were recorded for the stock solution stored in the dark, $5^{\circ} \mathrm{C}$.

Determination of total reducing capacity with Folin-Ciocalteu reagent. Folin-Ciocalteu reagent contains a mixture of phosphomolybdic and phosphotungstic acid complexes. The reagent reacts with phenols and nonphenolic reducing substances and forms chromogens which can be detected spectrophotometrically. The measured absorbance corresponds to the total reducing capacity. The total reducing capacity was determined in 1.9 and $0.95 \mathrm{mM}$ DCBQ aqueous solution stored at ambient temperature and exposured to natural light. Folin-Ciocalteu reagent (10 fold diluted in distilled water) was mixed with $\mathrm{H}_{2} \mathrm{O}$ and DCBQ stock solution in the volume ratio $10: 5: 1$, respectively. After a 3 -min incubation $1 \mathrm{~cm}^{3}$ of $20 \% \mathrm{Na}_{2} \mathrm{CO}_{3}$ was added and after further $15 \mathrm{~min}$ the absorbance was recorded in 1-cm quartz cuvette at 730 $\mathrm{nm}$ using a MARCEL MEDIA spectrophotometer (Singleton et al., 1999). Control measurement was done for 1.9-mM DCBQ stock solution stored in the dark, $5^{\circ} \mathrm{C}$.

Determination of DPPH scavenging activity. DPPH scavenging activity assay is commonly used for antioxidant activity determination in biological material (Gow-Chin Yen et al., 2002; Ting Sun et al., 2007). Here, 
this method was used as a modified procedure for an aqueous-ethanolic system.

DPPH scavenging activity of 1.9 and $0.95-\mathrm{mM}$ DCBQ solution, respectively was tested for $288 \mathrm{~h}$. DPPH scavenging activity was determined by the reduction of DPPH in aqueous-ethanolic system. Ethanolic solution $\left(1 \mathrm{~cm}^{3}\right)$ of $0.3 \mathrm{mM}$ DPPH was mixed with $1 \mathrm{~cm}^{3}$ of water and $0.2 \mathrm{~cm}^{3}$ of DCBQ solution. The decrease in absorbance in 1-cm quartz cuvette at $515 \mathrm{~nm}$ was measured in continuous mode for 26 min using a MARCEL MEDIA spectrophotometer. The radical scavenging ability towards DPPH was calculated from the following Eqn.:

$\%$ DPPH scavenging activity $=\frac{A_{\text {control }}-A_{\text {sample }}}{A_{\text {control }}} \times 100$

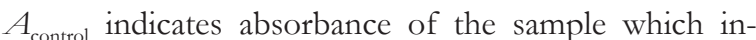
stead of DCBQ solution contained equal volume of water.

Control measurement was done for 1.9-mM DCBQ stock solution stored in the dark, $5^{\circ} \mathrm{C}$.

\section{RESULTS AND DISCUSSION}

\section{Kinetics of urease inhibition by $D C B Q$}

The inhibition progress curves as a dependence of the urease activity vs incubation time of urease with DCBQ are presented in Fig. 1. DCBQ inhibited urease in a time and concentration-dependent manner. Under the experimental conditions obeyed terms of pseudo-first-order reaction (excess of inhibitor over enzyme) urease was completely inactivated. This type of inhibition curves allowed to the use of Kitz-Wilson Eqn. (Eqn. 1) for kinetics analysis (Kitz \& Wilson, 1962; Silverman, 2002):

$1 / k_{\text {app }}=\left(K_{\mathrm{i}} / k_{\text {inact }}\right)\left(1 / c_{\text {inhibitor }}\right)+\left(1 / k_{\text {inact }}\right)$

where $k_{\text {app }}$ indicates a pseudo-first-order constant, $K_{\mathrm{i}}$ corresponds to a dissociation constant of enzyme-inhibitor intermediate complex, $c_{\text {inhibitor }}$ indicates inhibitor concentration and $k_{\text {inact }}$ is the rate constant of inactive enzyme formation:

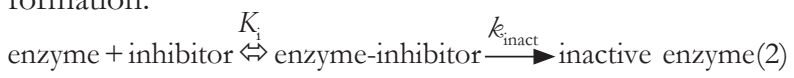

Transformation of the inactivation data into semilogarithmic scale (Fig. 1, inset) revealed that the reaction followed monophasic, pseudo-first-order kinetics. Linearization of the progress curves was a good approximation for curves obtained at high inhibitor concentrations. The curves obtained at the lowest inhibitor concentration studied $(4.8 \mu \mathrm{M})$ was linear until $30 \%$ of urease activity remained. The double reciprocal plot of the pseudo-firstorder constant $k_{\text {app }}$ as a function of DCBQ concentration is presented in Fig. 2. The system produced straight line passing through the origin $\left(R^{2}=0.99\right)$. This result indicated that inactivation expressed by Eqn. 2 in fact followed a simple bimolecular reaction:

urease $+\mathrm{DCBQ} \rightarrow$ inactive urease

The presence of intermediate complex urease-inactivator was undetectable because of the relatively fast inactivation compared with the urease-inactivator complex creation. The obtained second order rate constants $k^{\prime}\left(k^{\prime}=k_{\text {inact }} / K_{\mathrm{i}}\right)$ was equal to $0.057(\mu \mathrm{M} \mathrm{min})^{-1}$.

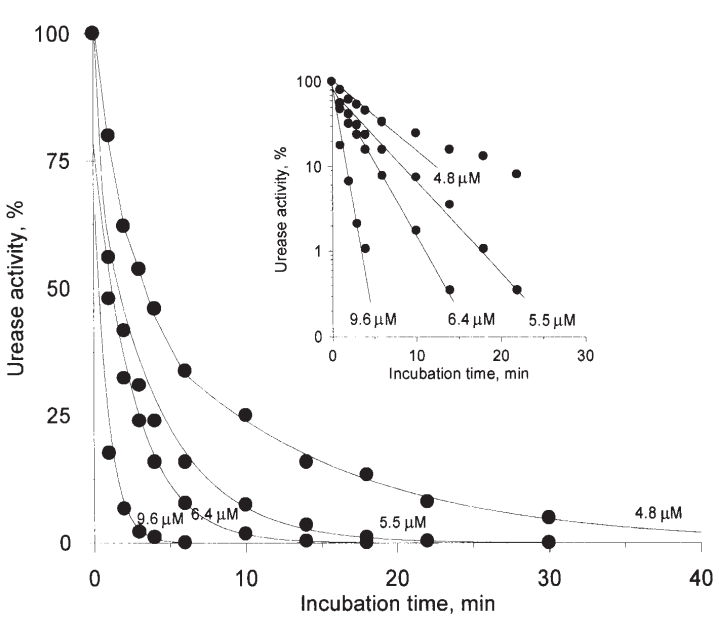

Figure 1. Urease inactivation progress curves as a dependence of enzyme activity vs incubation time for different DCBQ concentrations.

(Inset) Dependence of urease activity vs incubation time in semilogarithmic system. DCBQ concentrations are given numerically. Solutions of urease were mixed with solutions of DCBQ in volume proportion $1: 1$. After appropriate period of time, aliquots were withdrawn and urease activity was determined.

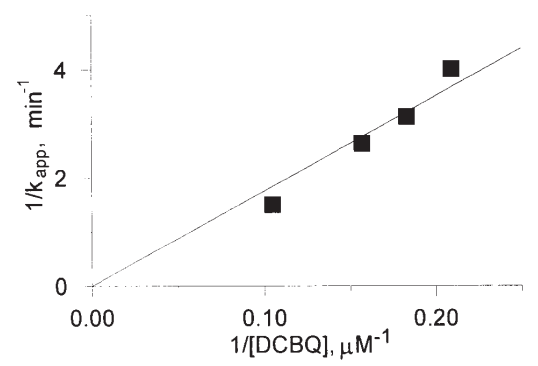

Figure 2. Dependence of $k_{\text {app }}$ vs DCBQ concentration in a double reciprocal system.

\section{Mechanism of urease inactivation by DCBQ. Thiol protective and reactivation experiments}

It was found that in the presence of $9.6 \mu \mathrm{M}$ DCBQ urease was completely inactivated after a 5-min incubation (Fig. 1). However, the presence of a thiol-protector such as L-cysteine, glutathione or dithiothreitol, at 0.25 $\mathrm{mM}$ completely prevented urease inactivation. This experiment indicated a crucial role of urease thiols (especially the active site cysteine residue) in the inhibition process. Chloroquinones are highly reactive towards nucleophiles hence DCBQ as the inhibitor follows the nucleophilic substitution with urease thiols (R-SH) according to Eqn. 4:<smiles>OC1CC(O)C(Cl)CC1O</smiles>

The process could lead to substitution of the subsequent chlorine. Such multisubstitution was observed for the urease inhibition by tetrachloro-1,4-benzoquinone (Zaborska et al., 2007). The presence of the thiol-protector in the incubation system allowed DCBQ to react with thiols of two origins: the urease thiols and the "free" thiol-protector. This rises the question: why did DCBQ prefer reaction with the "free" thiols rather than with the urease thiols? The first reason was the ratio 
of the thiol-protector to the urease thiols. The thiolprotector was used in a great excess over the DCBQ concentration. The ratio of urease thiols to DCBQ was much lower advantageous. However, the more important reason was unequal accessibility of the urease thiols for the inhibitor because of their different catalytic functions and structural positions in the molecule. Norris \& Brocklehurst (1976) determined the total thiol content in jack bean urease by 5,5'-dithiobis-(2-nitrobenzoic acid) (DTNB) titration in the presence of $6 \mathrm{M}$ guanidinum chloride. The experiment showed the presence of 15 thiol residues per enzyme subunit. However, only 6 of the 15 cysteines were accessible to the thiol reagent without denaturation of the enzyme. The difference in the quinones affinity towards various thiols was demonstrated in a previous article (Kot et al., 2010) for 2-hydroxy-1,4-naphthoquinone (lawsone). It was observed that lawsone reactivity with thiols followed the rule that the more complex thiol was, the less susceptible it was to the reaction. Lawsone showed an excellent reactivity with L-cysteine, much less with the tripeptide glutathione ( $\gamma$-Glu-Cys-Gly) and hardly reacted with urease.

The reactivation of DCBQ-inactivated urease was carried out by DTT application. Urease was incubated with DCBQ until the activity of the enzyme decreased to $0.9 \%$ (after a 5-min incubation), then DTT was added. Although DTT exhibited a protective effect on urease it failed to reverse the DCBQ inhibition (Fig. 3). The experiment was repeated for shorter time of urease incubation with DCBQ (less inhibited urease): 1 and 2 min. The results for all trials were consistent: urease did not regain its activity.

The quinone-modified urease would be susceptible to DT'T reactivation if the modification were a result of quinone-induced ROS action. ROS can oxidize the catalytic thiols to sulfenic and higher oxidized forms. Sulfenic acid can be reduced back to the initial thiol group by DT'T (Rodriguez et al., 2005). The DCBQ/urease system did not display such behaviour. This can be concluded as the lack of redox cycling contribution in DCBQ action on urease. Hence, the inactivation was caused by the direct substitution according to Eqn. 3. A similar mechanism was observed for tetrachloro-1,4-benzoquinone and tetrachloro-1,2-benzoquinone. These quinones inhibited urease also by the direct nucleophilic substitution irreversible in the presence of DTT (Kot et al., 2006). A complex mechanism of urease inhibition was shown by another chlorosubstitued quinone: 2,3-dichloro-1,4-naphthoquinone. This inhibitor modified urease

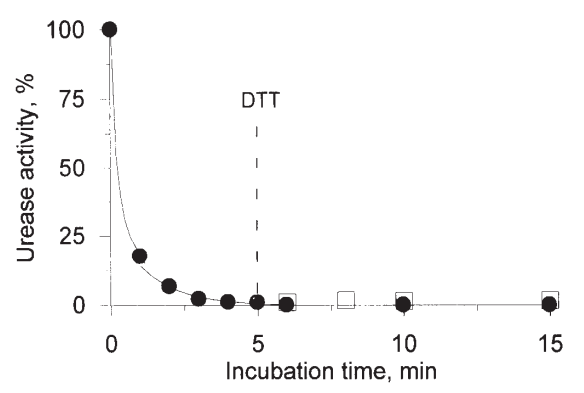

Figure 3. Reactivation of DCBQ-inactivated urease by DTT addition.

Activity of urease inactivated by DCBQ $(\bullet)$ and after DTT application ( $\square$ ). Urease was incubated with 5.6 $\mu \mathrm{M}$ DCBQ. After a 5-min incubation DTT was added to $50 \mu \mathrm{M}$. The activity of urease was determined before and after the DTT addition. After appropriate periods of time, samples of incubation mixture were withdrawn and urease activity was determined. by both mechanisms: nucleophilic substitution and ROS influence that resulted in partial reversibility of the inhibition (Zaborska et al., 2009).

\section{DCBQ decomposition in aqueous solution}

The decomposition of chlorosubstituted quinone in aqueous solution is an effect of at least two actions. The first one is hydrolysis. This process was well recognized for tetrachloro-1,4-benzoquinone (chloranil). In aqueous solution tetrachloro-1,4-benzoquinone undergoes two consecutive hydrolytic dechlorination reactions to yield chloranilic acid via the intermediate trichlorohydroxy-1,4benzoquinone. The hydrolysis is supposed to occur also in less chlorinated quinones (Sarr et al., 1995).

The next process responsible for transformation of quinone aqueous solution is photolysis. This process follows a complex mechanism which could be simplified to Eqn. 5:

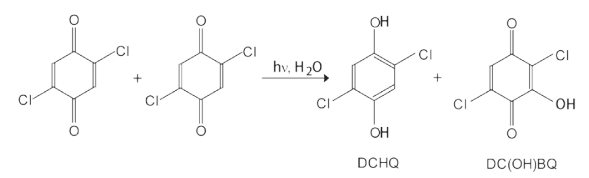

The products of DCBQ photolysis are 2,5-dichlorohydroquinone (DCHQ) and 2,5-dichloro-3-hydroxy1,4-benzoquinone (DC(OH)BQ) (Pochon et al., 2002). DCHQ as a hydroquinone derivative could be considered as a redox couple for DCBQ. The visible evidence of the quinone photolysis in aqueous solution is its colour change.

Visible spectra of DCBQ aqueous solution

Absorption spectra of $1.9 \mathrm{mM}$ DCBQ aqueous solution were registered for $288 \mathrm{~h}$ every $24 \mathrm{~h}$ or $48 \mathrm{~h}$ (at the beginning every few hours). Selected spectra are presented in Fig. 4. The spectra revealed that the most considerable changes concerned a broad band at $530 \mathrm{~nm}$. Until approximately $24 \mathrm{~h}$, this band exhibited increasing trend which later was reversed to a slow decreasing tendency. The time progress of absorbance at $530 \mathrm{~nm}$ is shown in Fig. 5B. The band at $530 \mathrm{~nm}$ was attributed to 2,5-dichloro-3-hydroxy-1,4-benzoquinone ( $\mathrm{DC}(\mathrm{OH})$ $\mathrm{BQ})$ formation. Then $\mathrm{DC}(\mathrm{OH}) \mathrm{BQ}$ underwent dimeri-

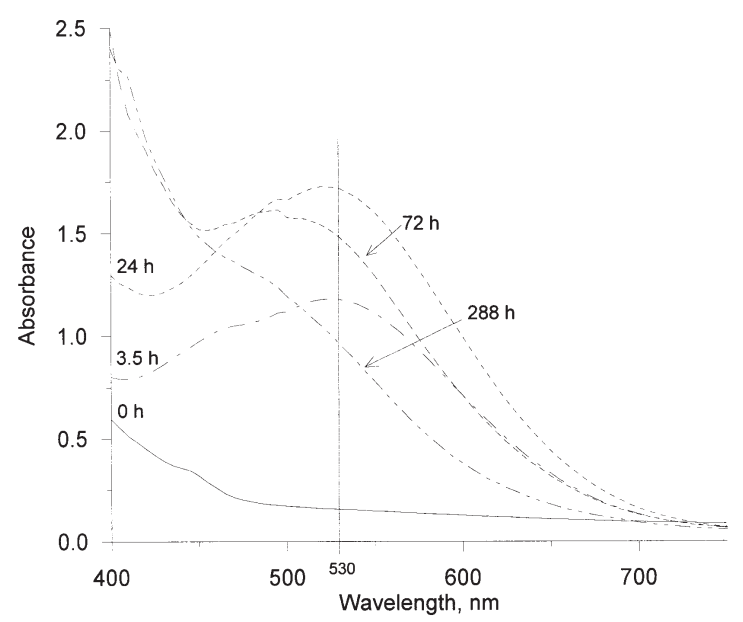

Figure 4. Absorption spectra of aqueous $1.9 \mathrm{mM}$ DCBQ solution exposured to natural light.

The spectra were recorded at different age of the solution as indicated on the graph. 

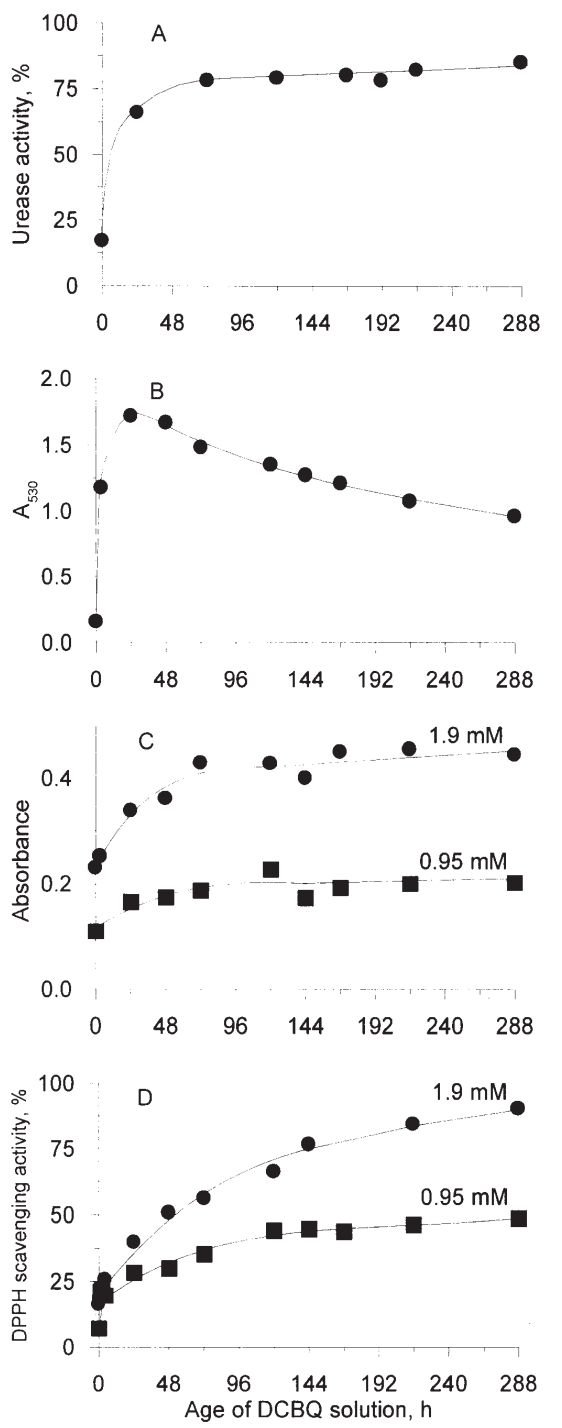

Figure 5. Changes of properties of DCBQ aqueous solution exposured to natural light vs age of the solution. The properties were monitored by application of different techniques:

(A) Dependence of urease activity inhibited by $6.5 \mu \mathrm{M}$ DCBQ vs age of DCBQ solution. (B) Dependence of absorbance at $530 \mathrm{~nm}$ $\left(A_{530}\right)$ of $1.9 \mathrm{mM} D C B Q$ vs age of $D C B Q$ solution. $A_{530}$ corresponds to the formation of 2,5-dichloro-3-hydroxy-1,4-benzoquinone. (C) Dependence of absorbance corresponding to the total reducing capacity of 1.9 or $0.95 \mathrm{mM}$ DCBQ determined by Folin-Ciocalteu method vs age of DCBQ solution. (D) Dependence of DPPH scavenging activity of 1.9 or $0.95 \mathrm{mM} D C B Q$ vs age of DCBQ solution.

sation that caused a decrease of the band. The bands related to dimer of $\mathrm{DC}(\mathrm{OH}) \mathrm{BQ}$, residual $\mathrm{DCBQ}$ and DCHQ were beyond the monitored wavelength range. These bands occupied coinciding region in the spectrum. Some evidence for the appearance of an increasing of an band below $400 \mathrm{~nm}$ was provided by the observation of increasing absorbance at the lower end of the spectra. Similar spectral changes assisted by HPLC identification were observed by Philipp et al. (1998) for resorcinol degradation.

The spectrum registered for the control DCBQ solution stored in the dark at low temperature did not exhibit any changes after $288 \mathrm{~h}$. This leads to the conclusion that the observed evolution of the DCBQ spectra was due to photolysis. Sarr et al. (1995) observed that tetrachloro-1,4-benzoquinone hydrolysis was promoted by al- kaline $\mathrm{pH}$. The neutral $\mathrm{pH}$ of our DCBQ stock solution did not support the process. Therefore, the contribution of hydrolysis to the conversion of DCBQ aqueous solution was negligible.

Influence of DCBQ decomposition in aqueous solution on urease inhibition

The change of the urease activity $v s$ age of the inhibitor solution is displayed in Fig. 5A. The loss of the DCBQ inhibitory power at the initial phase (approx. $48 \mathrm{~h}$ ) was fast. This fast phase was followed by a very slow decrease of the inhibitory effect with a tendency to attain the constant level at the residual urease activity of approx. $85 \%$. The first period of the weakening of the DCBQ solution inhibitory strength was well correlated with formation of $\mathrm{DC}(\mathrm{OH}) \mathrm{BQ}$ (Fig. 5B) that was associated with simultaneous decay of DCBQ in the system. The residual inhibitory power indicated that despite the photolysis DCBQ did not disappear totally. Therefore, urease was inhibited by app. $15 \%$ at the end of the monitoring time. The photoproduct DCHQ is unlikely to cause inhibitory effect since hydroquinone was not inhibitory active against urease (Bundy \& Bremner, 1973; Kot \& Zaborska, 2003). The next photoproduct $\mathrm{DC}(\mathrm{OH}) \mathrm{BQ}$ could slightly influence on urease activity because of the presence of hydroxysubstituent which significantly decreases the quinone inhibitory power (Bundy \& Bremner, 1973).

Influence of DCBQ decomposition in aqueous solution on total reducing capacity

The total reducing capacity was quantified as absorbance recorded with the use of spectrophotometric FolinCiocalteu method (more details in Materials and Methods). The change of the absorbance with the increasing age of DCBQ solution is presented in Fig. 5C. At the beginning, the absorbance increased significantly, later it remained constant. A similar course of the changes was found for both DCBQ concentrations studied. The progress of the absorbance corresponding to the total reducing capacity exhibited compatibility with the loss of DCBQ inhibitory power (Fig. 5A) and formation of $\mathrm{DC}(\mathrm{OH}) \mathrm{BQ}$ (Fig. 5B). According to Eqn. 4 the amount of photoproducts $\mathrm{DC}(\mathrm{OH}) \mathrm{BQ}$ and DCHQ were equal. DCHQ as a phenolic compound with a considerable reducing ability was considered to be the main compound responsible for the reducing capacity of the system.

Influence of DCBQ decomposition in aqueous solution on DPPH scavenging activity

The progress of DPPH radical scavenging activity vs age of DCBQ solution is depicted in Fig. 5D (DPPH radical scavenging activity is defined in Materials and Methods). The change of the DPPH scavenging activity was significantly more rapid during the first short period than in the following long one. The obtained results showed that for the beginning $48 \mathrm{~h}$ the DPPH scavenging activity increased by $25 \%$ and $48 \%$ in 0.95 and 1.9 $\mathrm{mM}$ DCBQ, respectively. The further $244 \mathrm{~h}$ resulted in the DPPH scavenging activity increase by $10 \%$ and $45 \%$ in 0.95 and $1.9 \mathrm{mM}$ DCBQ, respectively. The curve for 1.9 mM DCBQ solution showed a gradual increase of the DPPH scavenging activity while at $0.95 \mathrm{mM}$ the activity remained fairly constant after $48 \mathrm{~h}$. The main reason of the DPPH scavenging activity of the converted DCBQ solution was DCHQ. This compound was also 
responsible for the total reducing capacity. Unfortunately, the results obtained by both methods (Folin-Ciocalteu and DPPH) were only partly correlated. This inconsistency should be explained with relation to the complex mechanism of DCBQ phototransformation. Addition of DPPH radical to the DCBQ system probably provoked additional transformation of DCBQ resulting in extra $\mathrm{DPPH}$ scavenging. Therefore, DPPH use in the DCBQ system has to be analysed with its limitation. On the other hand, the gradually increasing DPPH scavenging activity (in spite of the constant inhibitory power and the almost constant total reducing ability) can be considered as an evidence of the presence of unphotolised DCBQ in the system. The incomplete DCBQ photolysis explains the incomplete loss of inactivation ability.

$\mathrm{DCBQ}$ in relation to other quinone urease inhibitors

Among the benzoquinones which have already been studied as urease inhibitors 2,5-dichloro-1,4-benzoquinone is located in the sequence:

1,4-benzoquinone $<2,5$-dichloro-1,4-benzoquinone $<$ tetrachloro1,4-benzoquinone

The sequence is arranged according to the increasing inhibitory power (Bundy \& Bremner, 1973; Zaborska et al., 2002; Kot \& Zaborska, 2006). The order is based on the values of inhibition constants and the concentration range affecting urease. The sequence proves that chlorination increases the inhibitory effect. Interestingly, DCBQ and tetrachloro-1,4-benzoquinone showed different modes of urease inhibition. Tetrachloro-1,4-benzoquinone revealed a bi-phasic inhibition with two intermediate complexes (Kot \& Zaborska, 2006; Zaborska et al., 2007) in contrast to DCBQ which showed a simple bimolecular inactivation (Eqn. 3). Both chlorobenzoquinones inhibited urease by the direct chlorine substitution with urease thiols (Eqn. 4). The higher chlorination of the tetrachloro-1,4-benzoquinone results in multiplication of the possible reactions: monochlorine substitution, multichlorine substitution with different enzyme molecules as well as with different thiols of that same enzyme molecule (quinone cross link) (Ommen et al., 1991; Ploemen et al., 1994). The higher reactivity and the different reaction opportunities of tetrachloro-1,4-benzoquinone could be the reason for the distinction between these chlorobenzoquinones as urease inhibitors. The difference in the DCBQ and tetrachloro-1,4-benzoquinone action has already been observed in relation to rat glutathione $S$-transferase 1-1 (GST) (Ommen et al., 1991; Ploemen et al., 1994). The effect of tetrachloro-1,4-benzoquinone was explained as an impact of non-specific reaction with GST thiol(s) and a specific modification of a catalytically involved residue.

\section{CONCLUSIONS}

DCBQ decomposition in aqueous solution results in a decrease of inhibitory ability and an increase of the total reducing activity as well as the DPPH scavenging activity. On the contrary to the light exposured DCBQ solution the DCBQ solution stored in the dark had its inhibitory strength as well as the total reducing activity and the DPPH scavenging activity unchanged. The lack of the conversion in the control DCBQ solution was also confirmed by unchanged visible spectrum. These findings indicate that the DCBQ conversion is through pho- tolysis not hydrolysis. The progress of the DCBQ photolysis resulted in production of DCHQ and $\mathrm{DC}(\mathrm{OH})$ $\mathrm{BQ}$. DCHQ as a phenolic compound was probably the main reducing agent in the system. Nevertheless, it was shown that the DCBQ photoproducts are less toxic than the parent compound which finding can have environmental significance. Moreover, since quinones are used as fungicides the obtained results could be helpful in their efficient dosage with respect to natural photodegradation. The findings may also have implications for waste treatment.

\section{REFERENCES}

Ashiralieva A, Kleiner D (2003) Polyhalogenated benzo- and naphthoquinones are potent inhibitors of plant and bacterial ureases. FEBS Lett 555: 367-370.

Benini S, Rypniewski WR, Wilson KS, Miletti S, Ciurli S, Mangani S (1999) A new proposal for urease mechanism based on the crystal structures of the native and inhibited enzyme from Bacillus pasteurii: why urea hydrolysis costs two nickels. Structure 7: 205-216.

Bolton JL, Trush MA, Penning TP, Dryhurst G, Monks TJ (2000) Role of quinones in toxicology. Chem Res Toxicol 13: 135-160.

Briton G (2006) The biochemistry of natural pigments. Cambridge University Press.

Bundy LG, Bremner JM (1973) Effects of substituted p-benzoquinones on urease activity in soils. Soil Biol Biochem 5: 847-853.

Dweek AC (2002) Natural ingredients for colouring and styling. Int J Cosmetic Sci 24: 287-302.

Gow-Chin Yen, Pin-Der Duh, Hui-Ling Tsai (2002) Antioxidant and pro-oxidant properties of ascorbic acid and gallic acid. Food Chem 79: 307-313.

Karplus PA, Pearson MA (1997) 70 Years of crystalline urease: what have we learned. Acc Chem Res 30: 330-337.

Kitz R, Wilson IB (1962) Esters of methanesulfonic acid as irreversible inhibitors of acetylcholinesterase. J Biol Chem 237: 3245-3249.

Kot M, Bicz A (2008) Inactivation of jack bean urease by N-ethylmaleimide: $\mathrm{pH}$ dependence, reversibility and thiols influence. J Enz Inh Med Chem 23: 514-520.

Kot M, Karcz W, Zaborska W (2010) 5-hydroxy-1,4-naphthoquinone (juglone) and 2-hydroxy-1,4-naphthoquinone (lawsone) influence on jack bean urease activity: elucidation of the difference in inhibition activity. Bioorg Chem 38: 132-137.

Kot M, Zaborska W (2003) Irreversible inhibition of jack bean urease by pyrocatechol. I Ens Inh Med Chem 18: 413-417.

Kot M, Zaborska W (2006) Inhibition of jack bean urease by tetrachloro-o-benzoquinone and tetrachloro-p-benzoquinone. J Enz Inh Med Chem 21: 537-542.

Kot M, Zaborska W, Juszkiewicz A (2000) Inhibition of jack bean urease by thiols. Calorimetric studies. Thermochim Acta 354: 63-69.

Krajewska B (2009) Ureases I. Functional, catalytic and kinetic properties: a review. I Mol Cat B 59: 9-21.

Lukens RJ (1971) Chemistry of Fungicidal Action. Molecular Biology, Biochemistry and Biophysics. vol 10, pp 62-89, Springer-Verlag, Berlin-Heidelberg-New York.

Mamiya G, Takishima K, Masakuni M, Kayumi T, Ogawa K, Sekita T (1985) Complete amino acid sequence of jack bean urease. Proc Jap Acad 61 Ser. B: $395-398$.

Mobley HLT, Hausinger RP (1989) Microbial ureases: significance, regulation, and molecular characterization. Microbiol Rev 531: 85-108.

Norris R, Brocklehurst K (1976) A convenient method of preparation of high-activity urease from Canavalia ensiformis by covalent chromatography and an investigation of its thiol groups with 2,2'-dipyridyl disulphide as a thiol titrant and reactivity probe. Biochem J 159: 245-257.

van Ommen B, Ploemen JHTM, Bogaards JJP, Monks TJ, Gau SS, van Bladeren PJ (1991) Irreversible inhibition of rat glutathione Stransferase $1-1$ by quinones and their glutathione conjugates. Biochem J 276: 661-666.

Ononye AI, Bolton JR (1986) Mechanism of photochemistry of p-benzoquinone in aqueous solutions. Optical flash photolysis studies. $J$ Phys Chem 90: 6270-6274.

Philipp B, Schink B (1998) Evidence of two oxidative reaction steps initiating anaerobic degradation of resorcinol (1,3-dihydroxybenzene) by the denitrifying bacterium Azoarcus anaerobius. I Bacteriol 180: 3644-3649.

Ploemen JH, Johnson WW, Jespersen S, Vanderwall D, van Ommen B, van der Greef J, van Bladeren PJ, Armstrong RN (1994) Activesite tyrosyl residues are targets in the irreversible inhibition of a class $\mathrm{Mu}$ glutathione transferase by 2-(S-glutathionyl)-3,5,6-trichloro1,4-benzoquinone. J Biol Chem 269: 26890-26897. 
Pochon A, Vaughan PP, Daqing Gan, Vath P, Blough NV, Falvey DE (2002) Photochemical oxidation of water by 2-methyl-1,4-benzoquinone: evidence against the formation of free hydroxyl radical. J Phys Chem A106: 2889-2894.

Rodriguez CE, Fukuto JM, Taguchi K, Froines J, Cho AK (2005) The interactions of 9,10-phenanthrenequinone with glyceraldehyde-3phosphate dehydrogenase (GAPDH), a potential site for toxic actions. Chem Biol Intern 155: 97-110.

Sarr DH, Chikoma Kazunga, Charles MJ, Pavlovich JG, Aitken MD (1995) Decomposition of tetrachloro-1,4-benzoquinone (p-chloranil) in aqueous solution. Environ Sci Technol 29: 2735-2740.

Silverman RB (2002) The organic chemistry of ensyme-catalyzed reactions. Revised edn, Appendix I: Enzyme kinetics, pp 584-587, Academic Press.

Singleton VL, Orthofer R, Lamuela-Raventos RM (1999) Analysis of total phenols and other oxidation substrates and antioxidants by means of Folin-Ciocalteu reagent. Methods Ensymol 299: 152-178.

Sirko A, Brodzik R (2000) Plant ureases: roles and regulation. Acta Biochim Pol 47: 1189-1195.
Ting Sun, Powers JR, Juming Tang (2007) Evaluation of the antioxidant activity of asparagus, broccoli and their juices. Food Chem 105: 101-106.

Weatherburn MW (1967) Phenol-hypochlorite reaction of determination of ammonia. Anal Chem 39: 971-974.

Zaborska W, Kot M, Bala A (2009) Kinetics of jack bean urease inhibition by 2,3-dichloro-1,4-naphthoquinone. Elucidation of the mechanism: redox cycling and sulfhydryl arylation. J Enz Inh Med Chem 24: 1082-1087.

Zaborska W, Kot M, Superata K (2002) Inhibition of jack bean urease by 1,4-benzoquinone and 2,5-dimethyl-1,4-benzoquinone. Evaluation of the inhibition mechanism. J Enz Inh Med Chem 17: 247-253.

Zaborska W, Krajewska B, Kot M, Karcz W (2007) Quinone-induced inhibition of urease. Elucidation of its mechanisms by probing thiol groups of the enzyme. Bioorg Chem 35: 233-242. 